\title{
Lineage Determination in the Immune System
}

\author{
Ellen V. Rothenberg \\ Division of Biology, California Institute of Technology, Pasadena, CA, USA
}

\begin{abstract}
Underlying all immune responses are the developmental programs that give immune cells their identities. Developmental controls determine the kinds of effector mechanisms that are available to different classes of cells, the degree of amplification that they can achieve through clonal expansion, the signals that they will respond to, and the limits that will be set on their abilities to alter function in different environmental conditions. As all these cell types are ultimately derived from common hematopoietic precursors, they each become distinct from other cell types at developmental choice points that define a time course of branching lineage relationships. This volume showcases striking new advances that illuminate the mechanisms governing several particularly interesting choice points where divergent immune cell identities are established.
\end{abstract}

\section{Recent perspectives on immune cell modes of development}

Development in the immune system raises a number of issues that are not usually addressed in classic embryonic developmental systems. One is the mobility of the cells, both in their functional roles and in their traffic to diverse inductive microenvironments, which introduces a stochastic element. Another is the question of when the irreversible aspects of immune-cell differentiation end and physiologically reversible 'effector responses' begin. The cells that mediate both adaptive and innate branches of immunity are specialized for the ability to mobilize finely tuned response programs based on the combinations of signals that they detect in the environment. This responsiveness involves major inducible changes in gene expression as well as effects on migration, morphology, and proliferation or death. All mature immune cells have sophisticated machinery for powerful signal-dependent responses. The behavior of a mature immune cell in a given situation can be seen as the superimposition of an environmentally modulated gene expression program on top of the core gene expression program dictated by that cell's developmental history. But what determines the stability of the core program?

How this actually works is turning out to be more interesting, and more complex, than might have been suspected earlier, as described by the reviews in this volume. There is an increasing appreciation of the highly branched nature of immune cell developmental pathways. The best known cases are the divergence of different T-cell receptor (TCR) $\alpha \beta$ Tcell subtypes during intrathymic positive selection, and the multiple T-helper (Th) subsets of conventional $\mathrm{CD}^{+}{ }^{+} \mathrm{TCR} \alpha \beta^{+} \mathrm{T}$ cells generated during responses to antigen. But multibranched pathways are now also recognized for TCR $\gamma \delta^{+}$cells, unconventional T cells, NK cells, and dendritic cells. It remains an active question how firm is the boundary between a 'lineage decision' and an 'activation state' in these branch points. Effector subsets of $\mathrm{CD} 4^{+} \mathrm{T}$ cells, such as Th1 and Th2 cells, were originally thought to be irreversibly polarized, terminal fates; however, as additional subsets have been defined, evidence has

Address for correspondence: Ellen V. Rothenberg, Division of Biology 156-29, California Institute of Technology, Pasadena, CA 91125 USA, Tel +1 626395 4992, Fax +1 626449 0756, evroth@ its.caltech.edu. 
also accumulated for plasticity and for the potential for interconversion among these effector fates.

Whereas different variants of T, NK, and dendritic cells are increasingly recognized as distinct, other branches of immune cell development appear now to be less clearly separated. It was thought for many years that the precursors of innate immune cells and the precursors of all adaptive immune cells were completely separate, having diverged much earlier in the hematopoietic hierarchy. This might suggest that once the initial lympho/myeloid decision was made, completely different sets of regulatory molecules could program the choices made at lower levels of the hierarchy. However, it is now clear that myeloid and lymphoid development can be closely intertwined, as discussed from several points of view in contributions in this volume (1-6). Not only can lymphoid and myeloid cells be derived from overlapping precursor subsets (under appropriate conditions) but also for developmental decisions, they can share use of the same lineage-defining transcription factors. These shared regulatory molecules participate in different combinations of factors to promote distinct cell fate choices across the adaptive/innate divide, such as PU.1 in dendritic cells and B cells, or Id2 and PLZF (Zbtb16) in NK cells, myeloid cells, iNKT cells, and some TCR $\gamma \delta$ cells $(4,7-10)$. It is the combination that specifically defines an 'adaptive' or 'innate' immune cell program, rather than any 'master gene' itself, and access to a cell fate can probably be restricted by control over any member of the combination.

\section{Organization and scope of this issue}

This volume highlights a number of key branchpoints in the lympho-hematopoietic developmental decision tree, where new evidence illuminates the molecules that exert control. T-lineage examples are prominent, as T-cell development has long been recognized to include multiple tiers of fate choices. Here these choices are discussed in the context of mechanisms used for B-cell, NK cell, dendritic cell, and progenitor cell fate determination as well. The reviews here cover lymphoid/myeloid lineage choice among hematopoietic precursors, B-cell developmental checkpoints, both in normal cells and as they are distorted in malignancy, specification of dendritic cells and divergence among dendritic cell subsets. A series of reviews then consider molecules that act at a succession of branchpoints along the T-cell developmental pathway: mechanisms separating T/NK precursors from myeloid cells, T from NK cells, $\alpha \beta$ T cells from $\gamma \delta$ T cells and different $\gamma \delta$ T cell subsets from one another, mechanisms creating divergences between conventional TCR $\alpha \beta^{+} \mathrm{T}$ cells and 'innate-like' iNKT cells, and finally mechanisms specifying and maintaining distinctions among different mature effector T-cell subsets. Focusing on the most mature and bestdefined cell types, contributors describe in detail the biochemical mechanisms of action of the controlling transcription factors themselves and the how they work on cis-regulatory elements of the key target genes in the differentiation pathways. These are models of the level of understanding that it is hoped the earlier, more complex developmental choices will eventually receive.

An area that is unfortunately underrepresented in this collection is the diversification and terminal maturation of B cells, which involves an important developmental switch that is based on the balance between two sets of mutually antagonistic regulators. However, the relationships between 'adaptive immune' lymphoid programs for B and T cells and the 'innate immune' differentiation programs used in dendritic cells, natural killer cells, monocytes, and granulocytes are a recurrent theme. Contributions in this volume address these relationships both at the cell biological level and in terms of the roles of factors like PU.1, E proteins, STAT factors, GATA-3, PLZF, Notch, ThPOK, T-bet, and the newly appreciated factor Bcl11b, as summarized below. 


\section{Early lymphoid precursors and the question of prior restriction}

$\mathrm{B}$ cells and $\mathrm{T}$ cells share undisputed central roles in adaptive immunity, also sharing many similar features of cell biology and the unique use of recombination-activating gene (RAG)mediated recombination to distinguish them from all other cell types, including even their lymphoid relatives the NK cells. The discovery of common lymphoid precursors marked by interleukin-7 receptor (IL-7R) expression (11) was a watershed for the field, as the first prospective isolation of individual progenitors with defined partial restrictions on potential, but it was also deeply appealing as evidence that the similarities between $\mathrm{T}$ and $\mathrm{B}$ cells might result from a uniquely shared inheritance. However, problems have arisen with the notion that $\mathrm{B}$ and $\mathrm{T}$ cells emerge from a precursor that first loses all nonlymphoid potentials before choosing between $\mathrm{T}$ and $\mathrm{B}$ fates.

These problems have commanded most attention with respect to the T-cell pathway, where isolation of an ordered succession of precursors from the thymus is fairly easy, and many independent labs have observed what these cells do when tested in different environmental conditions (reviewed here in 2, 3). It is seen here that B-cell potential is completely extinguished soon after the precursors enter the thymus, and yet a robust myeloid potential continues to be preserved for multiple cell divisions into T-cell specification. This myeloid potential is conditional, as it is restricted by environmental signals in the thymus, but it is quite evident upon transfer of the cells to permissive conditions in vitro, so that the later stage when myeloid potential is also lost can be clearly defined. There is also molecular evidence to explain how the normal gene regulatory features of early T-cell precursors in the thymus would still give access to myeloid but not B-cell programs. These T-lineage precursor assays thus imply that $\mathrm{T}$ and $\mathrm{B}$-cell potential segregate before $\mathrm{T}$-cell precursors lose myeloid or NK potential. However, evidence both old and new has been adduced to show that in vivo, under unperturbed conditions, the cells that seed the thymus may very well have a history of IL-7R expression, resembling the common lymphoid precursors. Even though these cells can undergo myeloid differentiation in vitro, they are poorer at generating myeloid cells in vivo than stem cells or dedicated myeloid precursors $(12,13)$, and the meaning of this discrepancy has been sharply debated.

The authors in this volume address this problem from several points of view. Yang et al. (2) review the observed sequence of lineage commitment stages for T-cell precursors, combining discussion of underlying transcriptional requirements with an emphasis on the environmental dependence of all these fate decisions. Thus, T-lineage competence depends first of all on the ability of the candidate precursors to enter the thymus, where the allimportant Delta-like 4 Notch ligand drives the inductive signaling that triggers and sustains the T-cell program. These authors point to the crucial importance of chemokine receptor regulation in controlling this process, thus determining the range of inductive signals a cell would ever actually see in vivo. Kawamoto et al. (3) focus on mechanisms that sustain and limit intrinsic myeloid competence, relating these to a broader hematopoietic context. They argue that a conditional myeloid potential may be a basic feature of hematopoiesis, a latent but useful program option with deep evolutionary roots that can be given up only when T, B, or erythroid cells become fully committed to another fate. Kondo (1) reviews the early lymphoid precursor debate from a vantage point based on B and myeloid-development, which both develop in the bone marrow. Here again, there is a conflict between the range of potentials that 'lymphoid precursors' show in vivo and in vitro. Kondo notes that in fact the bone marrow microenvironment is quite diversified and that regulated migration into particular zones within it is crucial for cells to receive appropriate signals for the lymphoid and myeloid programs. He cites results that inhibition of the appropriate chemokine receptor signals may be sufficient to reveal the latent myeloid potential in lymphoid precursors. Thus, even if lymphoid precursors express myeloid transcription factors, a strong bias for 
lymphoid lineage restriction may arise by programming cells to migrate in vivo to sites where lymphoid but not myeloid development is supported. Thus, the range of competence of the cells in vivo is partly a result of intrinsic regulatory state but also partly a result of the cells' ability to choose their own homing sites, where cell non-autonomous mechanisms then come forcefully into play.

\section{Lessons from B-cell and dendritic cell development}

B-cell developmental paradigms have led the field for over a decade (14-17). B cells depend for their specification on a small group of transcription factors, two of which, EBF1 and Pax5, are B-lineage specific, so that hematopoietic precursors lacking these factors show selective B-cell defects. It was through analysis of these factors' effects that the distinction first emerged clearly between positive regulation of lineage-specific genes ('specification') and exclusion of alternative fates ('commitment')(18). More widely shared factors like the E-protein E2A gain B-cell specific roles through incorporation in a network organized around the core of these two highly specific factors (19-21), as reviewed here by Zandi et al. (6). EBF1 and Pax5 and the expression of B-cell target genes in precursors are among the earliest signs of competence to generate B-cell progeny. Zandi et al. describe how lineage priming, i.e. initial onset of B-cell gene expression, substantially precedes commitment, and then the separate mechanisms through which actual B-lineage commitment is established.

One crucial regulator used in B-cell development is shared with myeloid cells, namely PU.1. This Ets-family transcription factor works not only to promote myeloid and dendritic cell development but also to set up a pool of competent precursors for lymphoid development, and much later, to regulate aspects of B-cell responses to antigen (4). Carotta et al. now review these innate and adaptive functions, highlighting a dose-dependent role for PU.1 in dendritic cell development that is explained in part by exquisitely sensitive PU.1 regulation of the Flt3 (Flk2) cytokine receptor gene.

Dendritic cells are highly diverse and programmed through distinctive, signal-dependent pathways. Watowich and Liu (22) reveal the web of mechanisms through which instructive signaling from cytokine and Toll-like receptors blends with cell-intrinsic transcription factor activities to direct dendritic cell subtype programs. In this complex network, there is clear evidence that distinct, cytokine-activated signal transducer and activator of transcription (STAT) factors and interferon-response factors (IRFs) play individualized, instructive roles both to foster certain fates and to block others. This recently appreciated network echoes aspects of the complex STAT-dependent networks governing $\mathrm{CD} 4^{+} \mathrm{T}$-cell effector fates, described later in the volume.

\section{Regulatory factors that define lymphocyte identity: early precursor, $T$, and NK fates}

PU.1 is perhaps the main transcription factor that all immune cells, adaptive and innate, most distinctively share, for at least some parts of their developmental histories. In contrast, the next sections of this volume focus on the factors whose activities cause the discontinuities between one immune cell type and another.

If there were a 'master regulator' for the genes that distinguish $\mathrm{B}$ and $\mathrm{T}$ cells from innate immune cells, E proteins would probably head the list of candidates. This family of transcription factors, including E2A, HEB (Tcf12), and E2-2 (Tcf4), repeatedly contributes to lymphoid developmental potential as well as to developmental subchoices and checkpoints within lymphoid fates. As reviewed by De Pooter and Kee (8), these factors and their intricately regulated inhibitors play roles in setting up precursors with lymphoid 
potential in the first place, blocking diversion to the myeloid mainstream, and again to separate B and T cells from their innate relatives like NK cells and most dendritic cells. However, constitutively activated effectors with hybrid innate/adaptive character, like iNKT cells (invariant TCR $\alpha$ NK-like T cells), also appear to use E protein inhibitors for their final maturation. De Pooter and Kee set the actions of $\mathrm{E}$ proteins in the context of the broader networks of regulatory genes in $\mathrm{B}$ and $\mathrm{T}$ cells.

For T cells, an equally indispensable factor is GATA-3. Hosoya et al. (23) review the complex activities of this factor, which is crucial for establishing the source pool of progenitor cells within the thymus and is then used again and again throughout T-cell development. Work from the authors' own laboratory as well as others illustrates the extreme dose dependence of GATA-3 in T-cell development from early stages, where it can act as a bridge between the T-cell fate and other fates for precursors, such as NK cells and even mast cells. These features of GATA-3 function have made it a challenge to study, but its vital role in T-cell development is now yielding to advanced transgenic and molecular approaches (23).

For T-lineage precursors, the most closely related non-T fate is that of NK cells. Although many NK cells develop without entering the thymus, there is substantial NK developmental potential among early T-cell precursors and a distinctive set of NK cells that develops in the thymus as well, and the regulatory inputs that determine these NK fates are reviewed by Klein Wolterink et al. (9). It has been known for a decade that E protein antagonist Id2 is important for NK cell maturation, but a number of other transcription factors besides GATA-3 and Id 2 are now clearly implicated in distinct aspects of NK cell development. Recently, the T-cell transcription factor Bcl11b has been identified as a factor that sets the boundary between 'T-cell' and 'NK cell' gene expression programs $(24,25)$, as well as a factor that controls a commitment checkpoint within the T-lineage itself $(25,26)$. Originally known to be required for correct regulation of later stages of T-cell development and selection (27-31), this newly recognized early role makes Bcl11b interesting for its role in Tlineage identity. Bcl11b features prominently in six articles in this collection $(2,3,5,8,9,32)$. The nature of this factor and the evidence for its role in NK development are reviewed in detail by P. Liu et al. (32). The evidence they discuss implies that Bcl11b may provide the single regulatory function that is crucial to distinguish the T-cell fate from the NK cell fate. These authors also describe the remarkable functional properties of mature $\mathrm{T}$ cells that have been converted to a partial NK-like status by deletion of Bcl11b in vivo. A model proposing that $\mathrm{T}$ vs. NK lineage choice decisions may be based on the opposition of Bcl11b to Nfil3 is presented by Klein Wolterink et al. (9).

The last of the reviews on early T-cell development, from the guest editor's group (5), draws on regulatory gene expression profiling and analysis of Bcl11b-deficient mutants to argue for a two-phase program in early T-cell development. A checkpoint boundary (see also 3 ) is proposed to divide an initial, growth promoting phase, which is governed by a legacy of stem-cell associated genes, from the later, post-commitment phase focused on TCR gene rearrangement and selection, when the stem-cell associated genes are repressed. In this model, the bridges from the T-cell program to alternative fates depend on the specific regulatory state in the initial phase. Bcl11b is sharply induced in vivo just before the boundary between the phases. It is required directly or indirectly to drive the loss of selfrenewal potential as well as to 'gate' the transition to commitment within the T-cell development program (5). 


\section{Regulation of distinct T-cell subtypes: divergent fates in the thymus}

The transition to commitment is closely linked to the earliest point of bifurcation between at least some of the T cells that can develop as TCR $\gamma \delta$ cells and those that will develop as TCR $\alpha \beta$ cells. The $\alpha \beta / \gamma \delta$ lineage divergence has become a focus of high attention in recent years, as TCR $\gamma \delta$ cells have been revealed to constitute a diverse cluster of cell types. TCR $\gamma \delta$ cells are distinct from each other in use of Notch signals, E proteins and their antagonists, and other regulatory factors including ThPOK (cKrox, Zbtb7b), T-bet (Tbx21), and its relative Eomesodermin, and Runx3, and also functionally distinct in their expression of the effector cytokines IFN $\gamma$ or IL-17, as reviewed here by Kreslavsky et al. (10). These authors consider the mechanisms that separate TCR $\gamma \delta$ differentiation pathways from TCR $\alpha \beta$ differentiation pathways as well as models for TCR $\gamma \delta$ subset selection, some of which appear to use highly biased selections of $\gamma$ and $\delta$ gene segments in their TCR rearrangements. Taking aim at the underlying problem of the nature of 'the TCR $\gamma \delta$ program', they challenge whether this is a single program with subordinate branches or whether it is in fact a collection of distinct regulatory programs.

The next major intrathymic bifurcation is the separation between $\mathrm{CD} 4^{+}$and $\mathrm{CD} 8^{+} \mathrm{TCR} \alpha \beta$ cells, as a result of positive selection. The discovery that ThPOK (cKrox, Zbtb7b) was a dominant and indispensable regulator of the $\mathrm{CD} 4^{+}$cell fate was one of the major advances in this field $(33,34)$. As for many of the factors involved in T-cell development, however, ThPOK is turning out to play a wider range of roles, and these are described in detail by Kappes (35). In particular, ThPOK is important for generation of two of the most mature subsets of TCR $\gamma \delta$ cells and to regulate the cytokine expression repertoire of the mature $\mathrm{TCR} \gamma \delta$ pool. ThPOK is also needed to confer or maintain some of the properties of iNKT cells.

The iNKT fate itself is a seeming hybrid, with much of an innate-like cell type in its response machinery in spite of its conventionally adaptive use of rearranging TCR $\alpha \beta$ genes, and its requirement for TCR ligand-dependent positive selection. There are different subsets of these cells as well and an increasingly rich appreciation of the roles they can play in immune responses. As reviewed by Das et al. (7), iNKT cells (and other innate-like T-cell subsets) have a distinctive set of positive selection signaling requirements and a distinctive requirement for the Zbtb factor PLZF (Zbtb16), as well as use of the E protein antagonist Id 2 for their terminal differentiation, which divide them from conventional $\mathrm{T}$ cells. However, like conventional $\mathrm{CD} 4^{+} \mathrm{T}$ cells they are diverse in function and phenotype, and Das et al. review the evidence that 'innate-type' TCR $\alpha \beta$ cells exemplified by iNKT cells may be sub-specialized comparably to conventional CD4 $4^{+} \mathrm{T}$ cells (7). Taking the work on NK, TCR $\gamma \delta$, and iNKT cells together, diversity of TCR, type of TCR, functional subspecialization, and even the use of a TCR at all are capable of being combined in different ways at the adaptive/innate immune system interface.

\section{Mechanisms of effector T-cell divergence in the periphery: gene network- level lockdown and epigenetic plasticity}

The last part of the volume delves into the sophisticated approaches that are now revealing how particular transcription factors and particular transcription factor target genes actually execute their roles in these effector fate decisions. Balasubramani et al. (36) focus on the cis-acting molecular mechanisms involved in activating expression of the IFN $\gamma$ and IL-17 genes, which distinguish not only Th1 and $\mathrm{Th} 17 \mathrm{CD} 4^{+}$conventional $\mathrm{T}$ cells but also subsets of mature TCR $\gamma \delta$ cells (35) and subsets of iNKT cells (7). Balasubramani et al. (36) identify the far-spread regulatory sequences that are now recognized to contribute to the opening of these loci and the transcription factors that act at each of these sites. They show how the 
accessibility of these cis-regulatory sites even in 'terminally differentiated' effector cells is still open to modulation under the influence of certain cytokine signaling pathways. Turning to another close view of Th1 cell specification, this time from the focal point of the transacting transcription factor, Miller and Weinmann (37) take the killer T-cell, NK-cell, and Th1-cell regulator T-bet (Tbx21) as a model for biochemical examination. They detail the intricate combination of positive and negative regulatory effects of this single factor in terms of the different particular chromatin remodeling factors that interact with it through distinct protein-protein interaction domains, enabling T-bet to activate or repress specific targets. This example illustrates much of what is likely to be happening at the protein level with other transcription factors as well in order to bring about the chromatin changes discussed by Balasubramani et al. (36).

These fine-scale analyses ultimately must connect into a broader picture of transcription factor mobilization to define different $\mathrm{CD} 4{ }^{+} \mathrm{T}$-cell effector subtypes, and a model for this larger network is comprehensively laid out at the end of this volume by Zhu and Paul (38). The summary presented seeks to account not only for the channeling of effector cells into Th1, Th2, Th17, regulatory T cell, T follicular helper, and Th9 'fates' but also for the cytokine-dependent plasticity that remains to enable a limited degree of interconversion in particular circumstances, examples of which are noted in both of the previous reviews. This gene network and its signaling-dependent inputs illustrates the reciprocal interactions between the transcription factors within the cells and the cytokine signals that the cells do or do not receive from their environments, in part depending on the regulated expression of cytokine receptors and signal transduction genes (38). It is this iterative set of regulatory relationships that shapes the effector response of mature TCR $\alpha \beta$ T cells in the periphery.

\section{Conclusions}

The reviews in this volume address only a fraction of the immune cell lineage commitment field, but they illustrate several central points. One is how the combination of particular signals from the environment collaborates with a cell's current transcription factor regulatory ensemble to promote development along a particular pathway or to exclude an alternative. Another point is that in general, many immune cell development pathways are profoundly branched and diversified even after major aspects of lineage commitment, at least including dendritic cell, NK cell, or T-cell fates. These developmental pathways genuinely appear to splay out toward distinct endpoints that are not simply more or less mature but in fact different 'mature' states. Regulatory explanations for the boundaries between these subtypes are rapidly emerging, and they appear to be mechanisms somewhat less rigid than the lineage exclusion mechanisms separating major immune cell classes and the mechanisms conferring transient response functions. However, 'diversification factors' like E proteins and their antagonists, PLZF, and even PU.1, can be used to separate fates within markedly different major cell lineages. Also emerging from these reviews is that in the T-related lineages at least, early development and later effector development make use of overlapping but reshuffled sets of transcription factors. The 'opportunistic' use of transcription factors to work in different pathways points to explanations for developmental roles not based on 'the' target genes of a given transcription factor but rather in the distinct set of sites to which that transcription factor is recruited in a given developmental context, through interactions with other factors.

These examples offer a gentle corrective to models of developmental choice that are based on assuming a rigid dichotomy between two opposing 'master regulators'. Comparing different pathways discussed in this volume, we can see development from hematopoietic stem cells making use of multiple combinations of transcription factors that need not always mutually repress but which can work either cooperatively or antagonistically in different 
contexts. The reviews in this volume offer a glimpse of the astonishing richness of functionally distinct cell types that can be generated as a result and the fluid and highly regulated ways they can be used in the immune response.

\section{Acknowledgments}

A debt of gratitude is due to all the authors who contributed their time and excellent insights to create the reviews in this volume. The ideas presented in this overview were developed with support from grants from the NIH, R01 CA90233, R33 HL89123, and RC2 CA148278 and from the Albert Billings Ruddock Professorship at Caltech.

\section{References}

1. Kondo M. Lymphoid and myeloid lineage commitment in mutipotent hematopoietic progenitors. Immunol Rev. 2010; 238

2. Yang Q, Bell JJ, Bhandoola A. T-cell lineage determination. Immunol Rev. 2010; 238

3. Kawamoto H, Ikawa T, Masuda K, Wada H, Katsura Y. A map for lineage restriction of progenitors during hematopoiesis: the essence of the myeloid-based model. Immunol Rev. 2010; 238

4. Carotta S, Wu L, Nutt SL. Surprising new roles for PU.1 in the adaptive immune response. Immunol Rev. 2010; 238

5. Rothenberg EV, Zhang J, Li L. Multilayered specification of the T-cell lineage fate. Immunol Rev. $2010 ; 238$

6. Zandi S, Bryder D, Sigvardsson M. Load and lock: the molecular mechanisms of B-lymphocyte commitment. Immunol Rev. 2010; 238

7. Das R, Sant'Angelo DB, Nichols KE. Transcriptional control of invariant NKT cell development. Immunol Rev. 2010; 238

8. de Pooter RF, Kee BL. E proteins and the regulation of early lymphocyte development. Immunol Rev. 2010; 238

9. Klein Wolterink RGJ, García-Ojeda ME, Vosshenrich CAJ, Di Santo JP. The intrathymic crossroads of $\mathrm{T}$ and NK cell differentiation. Immunol Rev. 2010; 238

10. Kreslavsky T, Gleimer M, Garbe AI, von Boehmer H. $\alpha \beta$ versus $\gamma \delta$ fate choice: counting the T-cell lineages at the branch point. Immunol Rev. 2010; 238

11. Kondo M, Weissman IL, Akashi K. Identification of clonogenic common lymphoid progenitors in mouse bone marrow. Cell. 1997; 91:661-672. [PubMed: 9393859]

12. Schlenner SM, Madan V, Busch K, Tietz A, Laufle C, Costa C, Blum C, Fehling HJ, Rodewald HR. Fate mapping reveals separate origins of $\mathrm{T}$ cells and myeloid lineages in the thymus. Immunity. 2010; 32:426-436. [PubMed: 20303297]

13. Serwold T, Ehrlich LI, Weissman IL. Reductive isolation from bone marrow and blood implicates common lymphoid progenitors as the major source of thymopoiesis. Blood. 2009; 113:807-815. [PubMed: 18927436]

14. Mandel EM, Grosschedl R. Transcription control of early B cell differentiation. Curr Opin Immunol. 2010; 22:161-167. [PubMed: 20144854]

15. Ramirez J, Lukin K, Hagman J. From hematopoietic progenitors to B cells: mechanisms of lineage restriction and commitment. Curr Opin Immunol. 2010; 22:177-184. [PubMed: 20207529]

16. Singh H, Pongubala JM, Medina KL. Gene regulatory networks that orchestrate the development of B lymphocyte precursors. Adv Exp Med Biol. 2007; 596:57-62. [PubMed: 17338175]

17. Busslinger M. Transcriptional control of early B cell development. Annu Rev Immunol. 2004; 22:55-79. [PubMed: 15032574]

18. Nutt SL, Heavey B, Rolink AG, Busslinger M. Commitment to the B-lymphoid lineage depends on the transcription factor Pax5. Nature. 1999; 401:556-562. [PubMed: 10524622]

19. Heinz S, et al. Simple combinations of lineage-determining transcription factors prime cisregulatory elements required for macrophage and B cell identities. Mol Cell. 2010; 38:576-589. [PubMed: 20513432]

20. Lin YC, et al. A global network of transcription factors, involving E2A, EBF1 and Foxo1, that orchestrates B cell fate. Nat Immunol. 2010; 11:635-643. [PubMed: 20543837] 
21. Treiber T, et al. Early B cell Factor 1 regulates B cell gene networks by activation, repression, and transcription- independent poising of chromatin. Immunity. 2010; 32:714-725. [PubMed: 20451411]

22. Watowich S, Liu YJ. Mechanisms regulating dendritic cell specification and development. Immunol Rev. 2010; 238

23. Hosoya T, Maillard I, Engel JD. From the cradle to the grave: activities of GATA-3 throughout Tcell development and differentiation. Immunol Rev. 2010; 238

24. Li P, et al. Reprogramming of T cells to natural killer-like cells upon Bcl11b deletion. Science. 2010; 329:85-89. [PubMed: 20538915]

25. Li L, Leid M, Rothenberg EV. An early T cell lineage commitment checkpoint dependent on the transcription factor Bcl11b. Science. 2010; 329:89-93. [PubMed: 20595614]

26. Ikawa T, et al. An essential developmental checkpoint for production of the T cell lineage. Science. 2010; 329:93-96. [PubMed: 20595615]

27. Kastner $\mathrm{P}$, et al. Bcl11b represses a mature $\mathrm{T}$-cell gene expression program in immature $\mathrm{CD} 4^{+} \mathrm{CD} 8^{+}$thymocytes. Eur J Immunol. 2010 in press.

28. Zhang S, et al. Antigen-specific clonal expansion and cytolytic effector function of CD8+ T lymphocytes depend on the transcription factor Bcl11b. J Exp Med. 2010; 207:1687-1699. [PubMed: 20660613]

29. Albu DI, et al. BCL11B is required for positive selection and survival of double-positive thymocytes. J Exp Med. 2007; 204:3003-3015. [PubMed: 17998389]

30. Inoue J, Kanefuji T, Okazuka K, Watanabe H, Mishima Y, Kominami R. Expression of TCR $\alpha \beta$ partly rescues developmental arrest and apoptosis of $\alpha \beta \mathrm{T}$ cells in $\mathrm{Bc} 111 \mathrm{~b}^{-/-}$mice. $\mathrm{J}$ Immunol. 2006; 176:5871-5879. [PubMed: 16670294]

31. Wakabayashi $Y$, et al. Bcl11b is required for differentiation and survival of $\alpha \beta$ T lymphocytes. Nat Immunol. 2003; 4:533-539. [PubMed: 12717433]

32. Liu P, Li P, Burke S. Critical roles of Bcl11b in T-cell development and maintenance of T-cell identity. Immunol Rev. 2010; 238

33. He X, et al. The zinc finger transcription factor Th-POK regulates CD4 versus CD8 T-cell lineage commitment. Nature. 2005; 433:826-833. [PubMed: 15729333]

34. Sun G, et al. The zinc finger protein cKrox directs CD4 lineage differentiation during intrathymic T cell positive selection. Nat Immunol. 2005; 6:373-381. [PubMed: 15750595]

35. Kappes DJ. Expanding roles for ThPOK in thymic development. Immunol Rev. 2010; 238

36. Balasubramani A, Mukasa R, Hatton RD, Weaver CT. Regulation of the Ifng locus in the context of T-lineage specification and plasticity. Immunol Rev. 2010; 238

37. Miller SA, Weinmann AS. Molecular mechanisms by which T-bet regulates T-helper cell commitment. Immunol Rev. 2010; 238

38. Zhu J, Paul WE. Peripheral CD4 ${ }^{+}$T-cell differentiation regulated by networks of cytokines and transcription factors. Immunol Rev. 2010; 238 